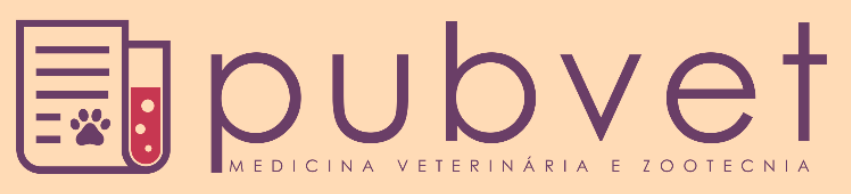

https://doi.org/10.31533/pubvet.v16n02a1033.1-6

\title{
Lesão de reabsorção dentária em onça-pintada (Panthera onca): Relato de caso
}

\author{
Marcos Vinicius Almeida Morais ${ }^{1 *}$, Dejoara de Angelis Zvoboda ${ }^{1 \oplus}$, Juliana Cavalli Santos ${ }^{1 \oplus}$, Helena \\ Baggio Soares $^{1 \oplus}$, Fernanda Taques Wendt ${ }^{2} \oplus$, Vivian Ferreira Rech ${ }^{2 \oplus}$, Rogério Ribas Lange ${ }^{3} \oplus$ \\ 'Laboratório de Odontologia Veterinária do Hospital Veterinário da Universidade Federal do Paraná. Curitiba, Paraná, Brasil. \\ ${ }^{2}$ Laboratório de Medicina Zoológica do Hospital Veterinário da Universidade Federal do Paraná. Curitiba, Paraná, Brasil. \\ ${ }^{3}$ Professor Doutor do Departamento de Medicina Veterinária, Universidade Federal do Paraná. Curitiba, Paraná, Brasil. \\ *Autor para correspondência, e-mail:marcos.v.a_morais@hotmail.com
}

Resumo. As lesões reabsortivas dentárias dos felinos (LRDF) são muito frequentes em gatos, sendo agravadas pelo avanço da idade do animal. É a doença oral mais comum dos gatos domésticos, sendo também relatada em leões, leopardos, onças, pumas, jaguatiricas e gatos-do-mato. Pode ser causa de dor oral intensa manifestando-se com hiporexia, sialorreia, "chattering" e sangramento oral. Apresentam-se clinicamente nas formas inflamatória e não inflamatória ou mista abrangendo as duas primeiras. Na forma não inflamatória pode acometer somente a raiz dentária não demonstrando sinais clínicos, sendo essa alteração visualizada nas imagens radiográficas como regiões radioluscentes na raiz dentária. Em animais silvestres cativos é recomendada a observação das instalações e a rotina dos animais, identificando precocemente sinais que possam indicar as doenças orais, como sangue e secreções em objetos que ele costuma morder, sinais de dor durante a mastigação e ingestão de líquidos, dificuldade de apreensão e escolha por alimentos mais macios. O exame completo da cavidade oral implica em anestesia geral, garantindo segurança para o animal e para a equipe envolvida no manejo. $\mathrm{O}$ objetivo deste relato é registrar um caso de lesão reabsortiva em onça-pintada e alertar sobre a importância do diagnóstico precoce de doenças orais em animais cativos, assim como o monitoramento constate, garantindo qualidade de vida.

Palavras-chave: Felídeos selvagens, lesão reabsortiva, odontologia veterinária.

\section{Dental resorption injury in jaguar (Panthera onca): Case report}

\begin{abstract}
Feline dental resorption lesions (FDRL) are very common in cats, being aggravated by advancing age of the animal. It is the most common oral disease of domestic cats, being also reported in lions, leopards, jaguars, pumas, ocelots and wild cats. It can be the cause of severe oral pain manifesting with hyporexia, drooling, chattering and oral bleeding. They present clinically in inflammatory and non-inflammatory or mixed forms, covering the first two. In the non-inflammatory form, it can affect only the tooth root, not showing clinical signs, and this change is seen on radiographic images as radiolucent regions in the tooth root. In captive wild animals, observation of the facilities and the routine of the animals is recommended, identifying early signs that may indicate oral diseases, such as blood and secretions on objects that it usually bites, signs of pain during chewing and ingestion of liquids, difficulty in apprehension and choice for softer foods. The complete examination of the oral cavity implies general anesthesia, ensuring safety for the animal and for the team involved in the management. The objective of this report is to register a case of reabsorptive lesion in a jaguar and warn about the importance of early diagnosis of oral diseases in captive animals, as well as constant monitoring, ensuring quality of life.
\end{abstract}

Keywords: Wild felids, reabsorptive lesion, veterinary dentistry 


\title{
Lesión por reabsorción dental en jaguar (Panthera onca): Reporte de caso
}

\begin{abstract}
Resumen. Las lesiones de reabsorción dental felina (LRDF) son muy comunes en los gatos y se agravan con el avance de la edad del animal. Es la enfermedad bucal más común de los gatos domésticos, siendo reportada también en leones, leopardos, jaguares, pumas, ocelotes y gatos salvajes. Puede ser la causa de un dolor bucal intenso que se manifiesta con hiporexia, babeo, "chaterring” y sangrado bucal. Se presentan clínicamente en formas inflamatorias y no inflamatorias o mixtas, cubriendo las dos primeras. En la forma no inflamatoria, puede afectar solo la raíz del diente, sin mostrar signos clínicos, y este cambio se ve en las imágenes radiográficas como regiones radiotransparentes en la raíz del diente. En animales silvestres cautivos, se recomienda observar las instalaciones y la rutina de los animales, identificando signos tempranos que puedan indicar enfermedades bucales, como sangre y secreciones en objetos que habitualmente muerde, signos de dolor al masticar e ingestión de líquidos, dificultad para aprehender y elegir alimentos más blandos. El examen completo de la cavidad bucal implica anestesia general, garantizando la seguridad del animal y del equipo involucrado en el manejo. El objetivo de este informe es registrar un caso de lesión reabsorbente en un jaguar y advertir sobre la importancia del diagnóstico temprano de enfermedades bucales en animales cautivos, así como el monitoreo constante, asegurando la calidad de vida.
\end{abstract}

Palabras clave: Felinos salvajes, lesión reabsorbente, odontología veterinaria

\section{Introdução}

A lesão reabsortiva dentária felina (LRDF) é uma das doenças da cavidade oral mais comum nos gatos, podendo apresentar-se na forma assintomática ou causar dor intensa reduzindo a qualidade de vida do animal. Caracteriza-se por erosões progressivas nos tecidos duros dentários como cemento, dentina e esmalte, por ação dos osteoclastos causando fraturas de coroa. Tem origem no ligamento periodontal por estímulo inflamatório constante (Gorrel, 2015; Roza \& Pinheiro, 2020; Wiggs \& Lobprise, 1997).

A etiologia da doença ainda não é bem esclarecida apesar das diversas teorias sobre o gatilho da lesão (Gorrel, 2015; Reiter et al., 2005; Reiter \& Mendoza, 2002; Wiggs \& Lobprise, 1997). Whyte et al. (2021) sugerem o aumento dos níveis séricos de creatinina e diminuição dos de ureia associados à presença de LRDF, indicando algum grau de lesão renal. A presença de lesão renal é possível quando há secreção constante de citocinas inflamatórias, que também pode estar presente com o avanço da idade do animal. Estudos também demonstram que o excesso na ingestão de vitamina D, presente em grande quantidade nas rações comerciais para gatos, está relacionada à presença de LRDF (Bellows et al., 2019; Reiter et al., 2005; Wiggs \& Lobprise, 1997). Animais de vida livre ou que recebem alimentação mista de caça e alimento comercial tem menores chances de desenvolver LRDF (Bellows et al., 2019; Wiggs \& Lobprise, 1997).

Os sinais clínicos observados são salivação intensa, sangramento oral, "chattering", gengivite acentuada, dificuldade de preensão e deglutição dos alimentos, hiporexia ou anorexia e halitose. "Chattering" é considerado o movimento de abertura de boca como se o animal estivesse conversando que indica dor oral (Roza \& Pinheiro, 2020). Como sinal patognomônico das lesões reabsortivas está a deposição de tecido inflamatório exuberante sobre a coroa, a fim de recobrir a erosão do meio externo (Bellows et al., 2019; Gorrel, 2015; Roza \& Pinheiro, 2020; Wiggs \& Lobprise, 1997). Também é comum encontrar acúmulo de cálculo dentário sobre a falha do esmalte devido à rugosidade do tecido dentário lesionado. Nos animais cativos é possível observar dificuldade na preensão dos alimentos e sensibilidade ao beber líquidos como sinais de dor (Fecchio et al., 2019).

As LRDF são classificadas tanto pelo grau de evolução quanto pela sua origem, sendo descritos quatro estágios quanto ao grau de evolução da lesão e três tipos quanto à sua origem conforme a tabela 1 (Reiter \& Mendoza, 2002; Roza \& Pinheiro, 2020).

Segundo a Associação Americana de Odontologia Veterinária, a prevalência chega a 70\% nos gatos domésticos e tem maior incidência com o avanço da idade (Roza \& Pinheiro, 2020). Animais cativos e 
de vida livre também podem ser acometidos pela LRD, sendo relatados casos em leões, leopardos, onças, pumas, jaguatiricas e gatos-do-mato (Berger et al., 1996; Fecchio et al., 2019). As lesões podem ocorrer em qualquer elemento dentário e em mais de um, geralmente de forma simétrica, mas há maior ocorrência nos dentes 307 e 407 (Gorrel, 2015; Wiggs \& Lobprise, 1997). Um estudo com 423 gatos demonstrou que somente radiografando os dentes pré-molares e molares mandibulares têm-se uma boa confiabilidade de triagem diagnóstica diminuindo o tempo anestésico e o nível de exposição à radiação (Heaton et al., 2004).

Tabela 1. Classificação das lesões de reabsorção dentárias.

\begin{tabular}{|c|c|}
\hline \multicolumn{2}{|l|}{ Grau de evolução } \\
\hline Estágio I & uando não há comprometimento da dentina, somente no esmalte ou cemento superficial. \\
\hline Estágio II & Quando as erosões invadem a dentina através do esmalte e do cemento. \\
\hline Estágio III & Quando comprometem a polpa dental. \\
\hline Estágio IV & Quando há perda da estrutura dentária, destruição da raiz e substituição dos tecidos causando anquilose. \\
\hline \multicolumn{2}{|l|}{ Origem } \\
\hline $\begin{array}{l}\text { Inflamatória ou Tipo } \\
\text { I radiográfico }\end{array}$ & $\begin{array}{l}\text { Restrito, na maioria das vezes, à coroa dental não havendo substituição do tecido. É sinal } \\
\text { patognomônico da lesão, exuberante tecido inflamatório sobre a erosão. Causa de dor intensa devido à } \\
\text { exposição pulpar. }\end{array}$ \\
\hline $\begin{array}{l}\text { Não-inflamatória ou } \\
\text { Anquilosante ou } \\
\text { Tipo II radiográfico }\end{array}$ & $\begin{array}{l}\text { Caracterizada pela substituição tecidual, causando anquilose dento-alveolar. Não há reação } \\
\text { inflamatória envolvida, portanto, esse tipo de lesão não causa dor. }\end{array}$ \\
\hline $\begin{array}{l}\text { Mista ou Tipo III } \\
\text { radiográfico }\end{array}$ & $\begin{array}{l}\text { epresenta as duas primeiras formas acontecendo concon } \\
\text { npossível distingui-las. }\end{array}$ \\
\hline
\end{tabular}

Fonte: Adaptado de Roza \& Pinheiro (2020).

O diagnóstico se dá tanto pelo exame clínico da cavidade oral por inspeção visual, como pela combinação de exame minucioso com sonda exploradora pontiaguda e radiografias intraorais (Gorrel, 2015). As radiografias intraorais são imprescindíveis para a observação das lesões uma vez que pode estar envolvida apenas a raiz dentária, não sendo possível a identificação no exame visual (Harvey \& Flax, 1992). Quando inspecionadas visualmente, somente são percebidas as lesões mais graves, que envolvem a exposição da câmara ou canal pulpar causando intensa dor (Bellows et al., 2019; Heaton et al., 2004; Lang et al., 2016; Wiggs \& Lobprise, 1997).

Existem três tipos de conduta para o tratamento das lesões reabsortivas dentárias: a exodontia, a amputação de coroa dentária e a conservativa. Nas duas últimas há necessidade de monitoramento a cada seis meses a um ano a fim de observar o avanço da lesão para a coroa. Se aplicam em casos nãoinflamatórios, quando somente a raiz dentária é acometida. A primeira conduta é a mais utilizada e consiste na extração total do elemento dentário, preferencialmente pela técnica aberta causando o menor trauma possível. O objetivo do tratamento é o alívio imediato da dor causada pela lesão e prevenção de sofrimento futuro, justificando a exodontia como técnica mais comumente realizada (Gorrel, 2015; Reiter \& Soltero-Rivera, 2014; Roza \& Pinheiro, 2020).

\section{Relato de caso}

Uma onça-pintada do Zoológico de Curitiba, com 11 anos de idade, macho inteiro, foi atendida no Hospital Veterinário da Universidade Federal do Paraná para exames de rotina e avaliação da cavidade oral. Foram coletadas amostras de sangue para hemograma e exames bioquímicos, também foi realizado ecocardiograma, eletrocardiograma, exame ultrassonográfico abdominal e coleta guiada de urina. Para exame físico e realização dos exames, o animal foi anestesiado com medicação pré-anestésica: dexmedetomidina $5 \mu \mathrm{g} / \mathrm{kg}$, metadona $0,3 \mathrm{mg} / \mathrm{kg}$, midazolam $0,1 \mathrm{mg} / \mathrm{kg}$ e cetamina $2 \mathrm{mg} / \mathrm{kg}$ com aplicação intramuscular e mantido em infusão endovenosa de propofol, remifentanil $10 \mu \mathrm{g} / \mathrm{kg} / \mathrm{h}$, dexmedetomidina $1 \mu \mathrm{g} / \mathrm{kg} / \mathrm{h}$ e cetamina $0,6 \mathrm{mg} / \mathrm{kg} / \mathrm{h}$.

No exame da cavidade oral foi observada retração gengival de $8 \mathrm{~mm}$ e bolsa periodontal de $15 \mathrm{~mm}$ em dente 104, bolsa periodontal de $12 \mathrm{~mm}$ em dente 204 e lesão de reabsorção com retração gengival e cálculo dentário grau III em dente 108 (Figura 1A). Foi realizada radiografia intraoral de toda a arcada dentária, sendo evidenciada lesão reabsortiva inflamatória em estágio II em dente 108 (Figura 1B). Foi decidido pela exodontia do dente com a lesão, mas devido ao tempo anestésico o procedimento odontológico foi remarcado para um segundo momento. 
Trinta e cinco dias após a avaliação diagnóstica, foi realizada a cirurgia oral. Como medicação préanestésica foi utilizada dexmedetomidina $5 \mu \mathrm{g} / \mathrm{kg}$ e metadona $0,3 \mathrm{mg} / \mathrm{kg}$ com aplicação intramuscular e mantido sob infusão endovenosa de propofol, remifentanil $10 \mu \mathrm{g} / \mathrm{kg} / \mathrm{h}$ e cetamina $0,6 \mathrm{mg} / \mathrm{kg} / \mathrm{h}$ durante o procedimento. A cavidade oral foi minuciosamente inspecionada com sonda exploradora pontiaguda, sendo realizado o tratamento da doença periodontal de todos os elementos dentários com raspagem do cálculo com caneta ultrassônica piezoelétrica e polimento com pasta profilática em caneta de contra ângulo de baixa rotação.

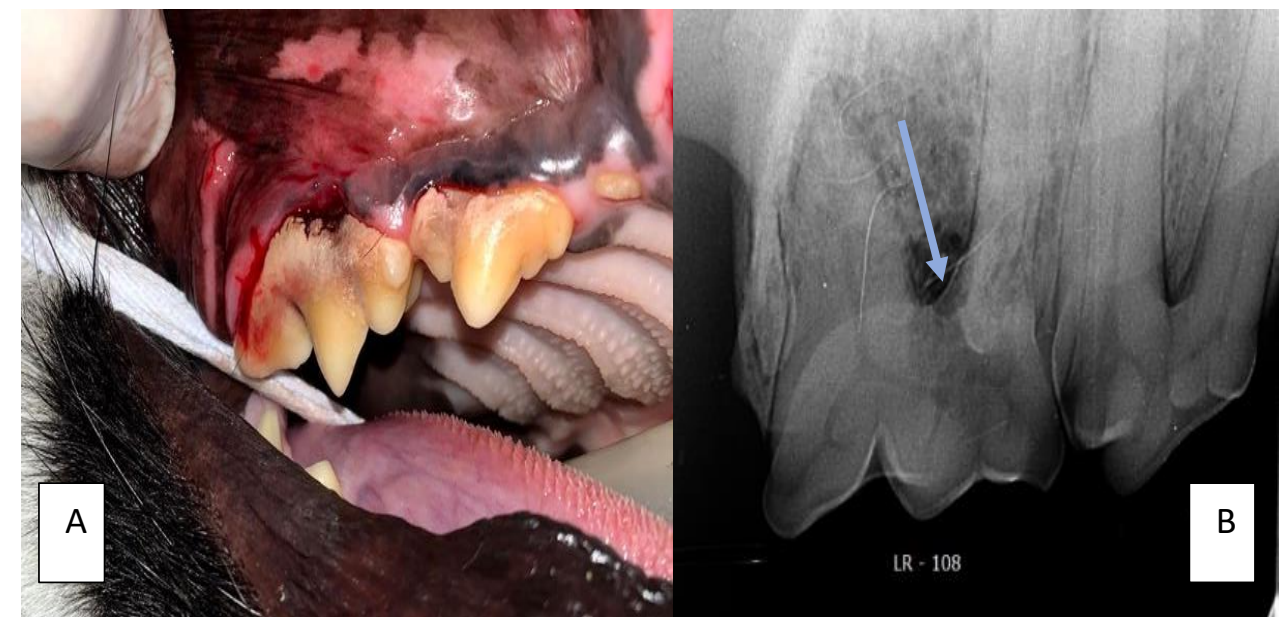

Figura 1. Aspectos da lesão reabsortiva em onça-pintada (Panthera onca). Na imagem A nota-se retração gengival, acúmulo de cálculo dentário grau III e sangramento à manipulação com sonda exploradora pontiaguda, em $\mathbf{B}$ a imagem radiográfica evidencia área de radioluscência próxima da furca (seta azul).

Após a limpeza da cavidade oral foi realizada a extração do dente 108. Foi efetuado bloqueio locoregional do nervo infraorbitário com $2 \mathrm{ml}$ de ropivacaína. Com lâmina de bisturi foi feito o retalho gengival (Figura 2A) e com broca diamantada em caneta de alta rotação o desgaste ósseo para a execução da técnica de exodontia aberta (Figura 2B). A odontosecção foi realizada com broca Zekrya em caneta de alta rotação separando o elemento dentário em três fragmentos. Os fragmentos radiculares foram extraídos com auxílio de alavancas luxadoras e boticão odontológico (Figura 2C). O retalho gengival foi suturado com pontos simples interrompidos com fio absorvível de poliglecaprone 4-0 (Figura 2D).

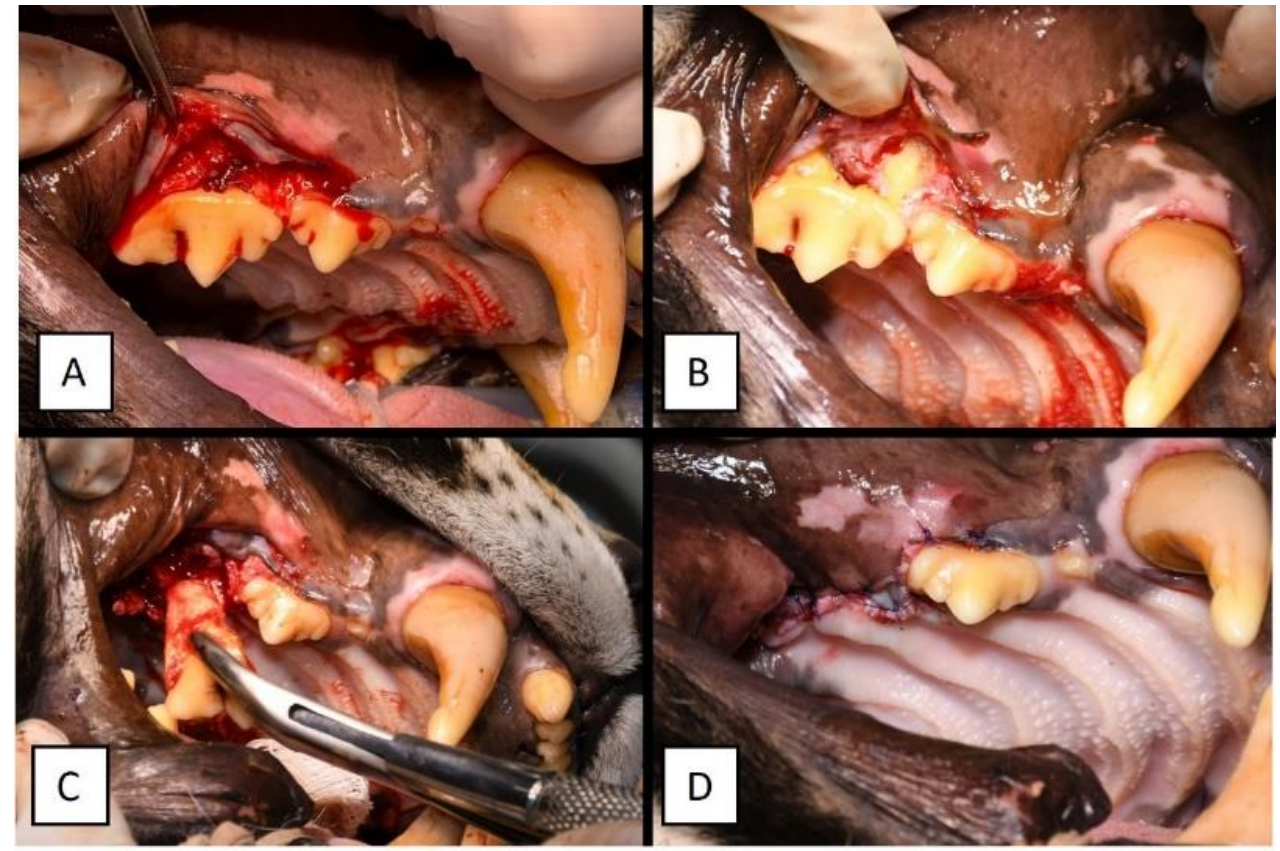

Figura 2. Aspectos da cirurgia oral em onça-pintada (Panthera onca): exodontia aberta do dente 108. Em A, retalho gengival para visualização do alvéolo e furca. Em B, desgaste alveolar com broca diamantada para realização da técnica de exodontia aberta. Em C, retirada de fragmento dentário após alavancagem. Em D, ferida cirúrgica suturada com fio absorvível de poliglecaprone 4-0. 
Como pós-operatório foi prescrita alimentação macia, Amoxicilina + ácido clavulânico $9 \mathrm{mg} / \mathrm{kg}$ duas vezes ao dia, durante 7 dias; Carprofeno $2 \mathrm{mg} / \mathrm{kg}$ uma vez ao dia, durante 3 dias e Tramadol $1 \mathrm{mg} / \mathrm{kg}$ uma vez ao dia, durante três dias. A recuperação anestésica e medicações pós-operatórias foram realizadas no parque Zoológico de Curitiba.

\section{Discussão}

A lesão de reabsorção dentária dos felinos é uma lesão erosiva dos tecidos duros dentários que pode expor a porção pulpar causando dor intensa. O sinal patognomônico da lesão na coroa dental é a deposição de tecido inflamatório acima da linha gengival, causando sensibilidade e sangramento oral. Geralmente as alterações são percebidas em estágio avançado, quando identificáveis no exame clínico. O diagnóstico se dá pelo exame com sonda exploradora pontiaguda do elemento dentário causando sangramento, e a confirmação definitiva pela imagem radiográfica intraoral (Heaton et al., 2004; Lang et al., 2016; Roza \& Pinheiro, 2020; Wiggs \& Lobprise, 1997). A lesão encontrada nesse relato foi classificada como inflamatória em estágio II, acometendo cemento, dentina e esmalte causando sensibilidade dentária. Ao exame da cavidade oral foi observado sangramento gengival e pulpar ao utilizar instrumento pontiagudo e radioluscência na região da furca dentária na radiografia intraoral.

As lesões tendem a ser simétricas, portanto, a monitoração frequente da saúde oral com auxílio de radiografias intraorais é fundamental para impedir o avanço da doença e a perda da qualidade de vida do animal (Gorrel, 2015; Wiggs \& Lobprise, 1997). A LRD relatada acometeu o dente quarto pré-molar maxilar, apesar de as lesões serem mais comumente encontradas nos pré-molares mandibulares (Gorrel, 2015). Uma vez que o dente 108 já foi acometido e, no momento do exame não foram percebidas alterações no dente contralateral 208, uma nova avaliação radiográfica é necessária no prazo de seis meses a um ano. Nos exames laboratoriais realizados não foram detectadas alterações nas concentrações séricas de creatinina ou ureia, não confirmando os dados prévios, mesmo com a idade avançada do animal (Whyte et al., 2021).

\section{Conclusão}

A lesão reabsortiva dentária dos felinos é bastante frequente em gatos domésticos e sempre deve ser investigada nos animais adultos. Por afetar a polpa dentária, pode causar dor intensa e diminuição da qualidade de vida do animal. Em felídeos selvagens, a avaliação radiográfica intraoral deve ser incluída na rotina a fim de investigar sua presença prevenindo o sofrimento do animal.

\section{Referências bibliográficas}

Bellows, J., Berg, M. L., Dennis, S., Harvey, R., Lobprise, H. B., Snyder, C. J., Stone, A. E. S., \& Van de Wetering, A. G. (2019). 2019 AAHA dental care guidelines for dogs and cats. Journal of the American Animal Hospital Association, 55(2), 49-69. https://doi.org/10.5326/JAAHA-MS-6933.

Berger, M., Schawalder, P., Stich, H., \& Lussi, A. (1996). Feline dental resorptive lesions in captive and wild leopards and lions. Journal of Veterinary Dentistry, 13(1), 13-21. https://doi.org/10.1177/089875649601300102.

Fecchio, R., Gioso, M. A., \& Bannon, K. (2019). Exotic animals oral and dental diseases. In H. D. Lobprise \& J. R. Dodd (Eds.), Wiggs's Veterinary Dentistry (p. 481). Wiley Online Library.

Gorrel, C. (2015). Tooth resorption in cats: pathophysiology and treatment options. Journal of Feline Medicine and Surgery, 17(1), 37-43. https://doi.org/10.1177/1098612X14560098.

Harvey, C. E., \& Flax, B. M. (1992). Feline oral-dental radiographic examination and interpretation. Veterinary Clinics of North America: Small Animal Practice, 22(6), 1279-1295. https://doi.org/10.1016/S0195-5616(92)50127-9.

Heaton, M., Wilkinson, J., Gorrel, C., \& Butterwick, R. (2004). A rapid screening technique for feline odontoclastic resorptive lesions. Journal of Small Animal Practice, 45(12), 596-601. https://doi.org/10.1111/j.1748-5827.2004.tb00181.x.

Lang, L. G., Wilkinson, T. E., White, T. L., Farnsworth, R. K., \& Potter, K. A. (2016). Computed tomography of tooth resorption in cats. Veterinary Radiology \& Ultrasound, 57(5), 467-474. https://doi.org/10.1111/vru.12387. 
Reiter, A. M., Lewis, J. R., \& Okuda, A. (2005). Update on the etiology of tooth resorption in domestic cats. Veterinary Clinics: Small Animal Practice, 35(4), 913-942. https://doi.org/10.1016/j.cvsm.2005.03.006.

Reiter, A. M., \& Mendoza, K. A. (2002). Feline odontoclastic resorptive lesions: an unsolved enigma in veterinary dentistry. Veterinary Clinics: Small Animal Practice, 32(4), 791-837. https://doi.org/10.1016/S0195-5616(02)00027-X.

Reiter, A. M., \& Soltero-Rivera, M. M. (2014). Applied feline oral anatomy and tooth extraction techniques: an illustrated guide. Journal of Feline Medicine and Surgery, 16(11), 900-913. https://doi.org/10.1177/1098612X14552365.

Roza, M., \& Pinheiro, F. (2020). Lesão de reabsorção dentária dos felinos. In M. Roza \& F. Pinheiro (Eds.), Manual de Odontologia Felina.

Whyte, A., Tejedor, M. T., Whyte, J., Monteagudo, L. V., \& Bonastre, C. (2021). Blood parameters and feline tooth resorption: A retrospective case control study from a Spanish University Hospital. Animals, 11(7), 2125. https://doi.org/10.3390/ani11072125.

Wiggs, R. B., \& Lobprise, H. B. (1997). Veterinary Dentistry: principles and practice. Lippincott-Raven Publishers.

\section{Histórico do artigo:}

Recebido: 1 de outubro de 2021

Aprovado: 28 de outubro de 2021

Disponível online: 22 de fevereiro de 2022
Licenciamento: Este artigoé publicado na modalidade Acesso Aberto sob a licença Creative Commons Atribuição 4.0 (CC-BY 4.0), a qual permite uso irrestrito, distribuição, reprodução em qualquer meio, desde que o autor e a fonte sejam devidamente creditados. 